\title{
How to derive priorities in AHP: a comparative study
}

\author{
Alessio Ishizaka ${ }^{a}$, Markus Lusti ${ }^{\mathrm{b}}$ \\ ${ }^{\text {a }}$ Audencia Nantes, Grande Ecole de Management, 8 route de la Jonelière, \\ 44312 Nantes Cedex 3, France \\ email: alessio.ishzaka@unibas.ch \\ ${ }^{\mathrm{b}}$ University of Basel, WWZ/Wirtschaftsinformatik, Petersgraben 51, 4051 Basel, \\ Switzerland \\ email: Markus.Lusti@unibas.ch
}

\begin{abstract}
A heated discussion has arisen over the "best" priorities derivation method. Using a Monte Carlo simulation this article compares and evaluates the solutions of four AHP ratio scaling methods: the right eigenvalue method, the left eigenvalue method, the geometric mean and the mean of normalized values. Matrices with different dimensions and degree of impurities are randomly constructed. We observe a high level of agreement between the different scaling techniques. The number of ranking contradictions increases with the dimension of the matrix and the inconsistencies. However these contradictions affect only close priorities.
\end{abstract}

KEYWORDS: Multi-Criteria Decision Making, simulation, AHP, eigenvalue, geometric mean 


\section{Introduction}

Since AHP was introduced by Saaty (Saaty 1977, 1980), it has been applied in numerous situations with impressive results (see lists in Golden, 1989; Shim, 1989; Vargas, 1990; Zahedi, 1986). AHP has been also criticised in the literature on account of weaknesses in its theoretical foundation. The three most important issues are the rank reversal problem (Johnson, et al., 1979), the priorities derivation method (Barzilai, et al. 1987, 2001b) and the comparison scale (Barzilai 2002, 2001a, 2001b, 1998; Salo et al., 1997). This paper focuses only on the priorities derivation methods and its consequences on rank contradictions. The literature covers several derivation methods which can be divided in two groups (Golany, et al., 1993): the eigenvalue approach and the methods minimizing the distance between the userdefined matrix and the nearest consistent matrix.

Among the eigenvalue methods, we distinguish the principal right eigenvalue (Saaty 1977, 1980), the principal left eigenvalue (Johnson, et al., 1979), and the modified eigenvalue method. Because of the reciprocity of the matrix, the last utilizes only the upper triangle to calculate the priorities. Unfortunately, since the ranking depends on the order of the alternatives in the matrix (Cogger, et al., 1985; Takeda, et al., 1987) the modified eigenvalue method is not reliable.

A minimum distance can be reached by different metrics. This has lead to the development of different derivation methods, in particular the logarithmic least squares also called geometric mean (Crawford, 1985), the least squares (Jensen, 1984), the weighted least squares (Chu, et al., 1979; Blankmeyer, 1987), and the logarithmic least absolute values (Cook, 1988).

With the exception of the logarithmic least squares equation, the methods are difficult to apply. In particular, the least squares method can result in several minima which make the choice ambiguous. Saaty (1984a) gives an example where the least squares method produces an illogical result.

A heated discussion has arisen over the "best" method. One side supports the eigenvalue method (Saaty, et al., 1984a; Saaty, et al., 1984b; Harker, et al., 1987; Saaty, 2001a; Saaty, 2003), the other side argues for the geometric mean (Barzilai, et al., 1987; Barzilai, et al., 1990; Barzilai, 1997; Barzilai, 2001). This dispute seems to be futile because experimental studies (Budescu, et al., 1986; Golany, et al., 1993) show that each method is best in at least one criterion (usually among the criteria it explicitly seeks to optimize), but neither is optimal by all or even most criteria.

The first part of the paper reviews theoretically the four derivation methods: the right eigenvalue method, the left eigenvalue method, the geometric mean and the mean of the normalised values. Next we describe a Monte Carlo simulation comparing the methods, and finally we evaluate these results. 


\section{Derivation of priorities}

A pairwise comparison matrix is called consistent if the transitivity (1) and the reciprocity (2) rules are respected.

$$
\begin{aligned}
& a_{i j}=a_{i k} \cdot a_{k j} \\
& a_{i j}=\frac{1}{a_{j i}} \quad \text { where } i, j \text { and } k \text { are any alternatives of the matrix }
\end{aligned}
$$

In a perfectly consistent matrix (3), all the comparisons $a_{i j}$ obey the equality $a_{i j}$ $=\frac{p_{i}}{p_{j}}$, where $p_{i}$ is the priority of the alternative $i$. Each method introduced in section 1 calculates identical priorities for consistent matrices. When the matrix contains inconsistencies, two approaches can be applied: the perturbation theory and the distance minimization.

$$
\mathbf{A}=\begin{array}{|c|c|c|c|c|}
\hline \mathrm{p}_{1} / \mathrm{p}_{1} & \ldots & \mathrm{p}_{\mathrm{1}} / \mathrm{p}_{\mathrm{j}} & \ldots & \mathrm{p}_{\mathrm{i}} / \mathrm{p}_{\mathrm{n}} \\
\hline \ldots & 1 & \ldots & \ldots & \ldots \\
\hline \mathrm{p}_{\mathrm{i}} / \mathrm{p}_{1} & \ldots & 1 & \ldots & \mathrm{p}_{\mathrm{i}} / \mathrm{p}_{\mathrm{n}} \\
\hline \ldots & \ldots & \ldots & 1 & \ldots \\
\hline \mathrm{p}_{\mathrm{n}} / \mathrm{p}_{1} & \ldots & \mathrm{p}_{\mathrm{n}} / \mathrm{p}_{\mathrm{i}} & \ldots & \mathrm{p}_{\mathrm{n}} / \mathrm{p}_{\mathrm{n}} \\
\hline
\end{array}
$$

\subsection{Mean of normalized values}

This is the oldest method and is based on three steps (example 1):

1. Sum of the elements of the column $j$

2. Normalization of the column $j$

3. Mean of row $i$

Demonstration :

All the elements of the column $j$ of the matrix (3) are summed, which gives

$$
\frac{p_{1}}{p_{j}}+\ldots+\frac{p_{i}}{p_{j}}+\ldots+\frac{p_{n}}{p_{j}}=\frac{\sum_{i=1}^{n} p_{i}}{p_{j}}
$$

The normalised value is calculated by dividing the comparison $a_{i j}=\frac{p_{i}}{p_{j}}$ by (4): 


$$
\frac{\frac{p_{i}}{p_{j}}}{\frac{\sum_{i=1}^{n} p_{i}}{p_{j}}}=\frac{p_{i}}{p_{j}} \cdot \frac{p_{j}}{\sum_{i=1}^{n} p_{i}}=\frac{p_{i}}{\sum_{i=1}^{n} p_{i}}
$$

The priority $i$ is the average of the elements (5) of the row $i$.

$$
\left(\frac{p_{i}}{\sum_{i=1}^{n} p_{i}}+\ldots+\frac{p_{i}}{\sum_{i=1}^{n} p_{i}}\right) \cdot \frac{1}{n}=\frac{n \cdot p_{i}}{\sum_{i=1}^{n} p_{i}} \cdot \frac{1}{n}=\frac{p_{i}}{\sum_{i=1}^{n} p_{i}}
$$

The priority $p_{i}$ is as expected the normalised priority $p_{i}$ normalised.

For this method no mathematical approach is available for dealing with inconsistent matrices.

\section{Example 1:}

Consider the following comparison matrix:

\begin{tabular}{|c|c|c|}
\hline 1 & 6 & 3 \\
\hline $1 / 6$ & 1 & $1 / 2$ \\
\hline $1 / 3$ & 2 & 1 \\
\hline
\end{tabular}

The method "mean of normalized values" derives the priorities as follow:

1. Add the elements of the columns: $\quad(1.5,9,4.5)$

2. Normalize the columns:

\begin{tabular}{|l|l|l|}
\hline 0.67 & 0.67 & 0.67 \\
\hline 0.11 & 0.11 & 0.11 \\
\hline 0.22 & 0.22 & 0.22 \\
\hline
\end{tabular}

3. Calculate the mean of the rows: $\quad(0.67,0.11,0.22)$.

\subsection{The eigenvalue approach}

Saaty $(1977,1980)$ proposes the principal eigenvector $\vec{p}$ as the desired priorities vector. It is calculated with the following equation:
$\mathbf{A} \cdot \vec{p}=\lambda \cdot \vec{p}$
where
A is the comparison matrix
$\vec{p}$ is the priorities vector
$\lambda$ is the maximal eigenvalue. 
Demonstration:

We multiply the consistent matrix (3) by the priority vector $\mathbf{p}=\left(p_{1}, \ldots, p_{i}, \ldots, p_{n}\right)$, we obtain:

$$
\mathbf{A} \mathbf{p}=n \mathbf{p} \quad \text { where } \begin{aligned}
& \mathbf{p} \\
& =\left(p_{1}, \ldots, p_{j}, \ldots, p_{n}\right) \\
& n=\operatorname{dimension} \text { de } \mathbf{A}
\end{aligned}
$$

Saaty $(1977,1980)$ justifies the eigenvalue approach for slightly inconsistent matrices with the perturbation theory, which says that slight variations in a consistent matrix imply slight variations of the eigenvector and the eigenvalue (example 2).

\section{Example 2:}

Figure 1 represents the characteristic equation of the consistent matrix A. The maximal eigenvalue is 3 (dimension of the matrix) and the associated eigenvector is $\vec{p}=(0.67,0.11,0.22)$. Figure 2 draws the characteristic equation of the near consistent matrix B, slightly modified from the matrix A. We can see that the characteristic equation, the eigenvalues and the priorities are also slightly modified.

The eigenvalue method is less transparent than the minimization of the distance. Many authors have underlined the unclearness of the eigenvalue process (Johnson, et al., 1979; Chu, et al., 1979). To clarify the eigenvalue method we apply the power method, a numerical method to calculate the maximal eigenvector (e.g. Lusti, 2002).

The power method relies on an iterative process (example 3), where:

1. The pairwise matrix is squared.

2. The row sums are then calculated and normalised. This is the first approximation of the eigenvector.

3. Using the resulting matrix. Step 1 and 2 are repeated.

4. Step 3 is repeated until the difference between these sums in two consecutive priorities calculations is smaller than the stop criterion.

consistent

$A=$\begin{tabular}{|c|c||c|}
\hline 1 & 6 & 3 \\
\hline $1 / 6$ & 1 & $1 / 2$ \\
\hline $\mathbf{1} / \mathbf{3}$ & 2 & 1 \\
\hline
\end{tabular} near consistent

B =

\begin{tabular}{|c|c|c|}
\hline 1 & 6 & $\mathbf{2}$ \\
\hline $1 / 6$ & 1 & $1 / 2$ \\
\hline $\mathbf{1} / \mathbf{2}$ & 2 & 1 \\
\hline
\end{tabular}




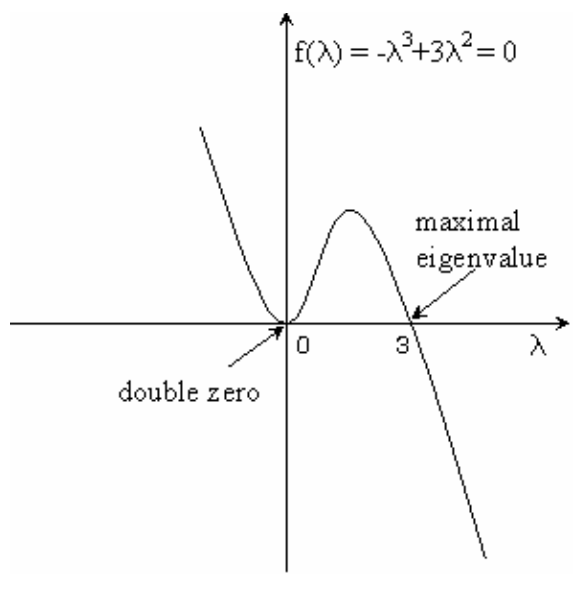

$$
\vec{p}=(0.67,0.11,0.22)
$$

Figure 1: Characteristic equation of the consistent matrix A

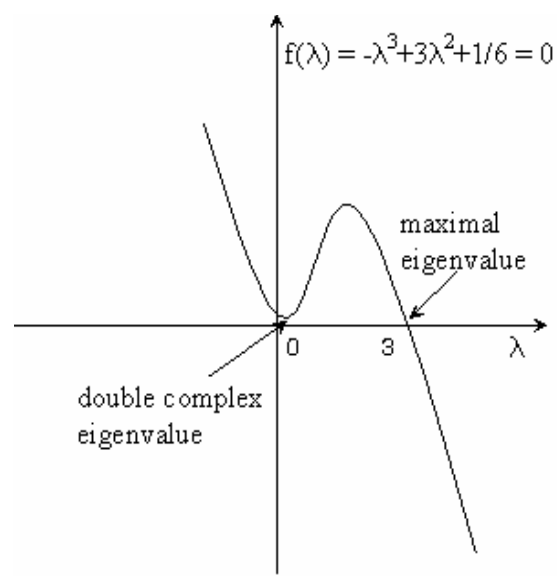

$$
\vec{p}=(0.61,0.12,0.27)
$$

Figure 2: Characteristic equation of the near consistent matrix B

Example 3:

Considering the inconsistent matrix $\mathrm{B}$ of example 2 , the priorities are derived as follow:

1. Square the matrix

\begin{tabular}{|c|c|c|}
\hline 3 & 16 & 7 \\
\hline 0.583 & 3 & 1.333 \\
\hline 1.333 & 7 & 3 \\
\hline
\end{tabular}

2. Sum and normalise the rows: $\quad(0.615,0.116,0.268)$.

3. Repeat step 1 and 2: $\quad(0.614,0.117,0.268)$.

4. Stop if the difference between the priorities of steps 2 and 3 is smaller than the stop criterion.

In example 3 , the value $a_{13}=7$ is the sum of:

$$
\begin{aligned}
& a_{13}=a_{11} \cdot a_{13}=1 \cdot 2=2 \\
& a_{13}=a_{12} \cdot a_{23}=6 \cdot \frac{1}{2}=3 \\
& a_{13}=a_{13} \cdot a_{33}=2 \cdot 1=2
\end{aligned}
$$

These three lines are the direct and indirect comparisons deduced by the transitivity rule (1). Since the matrix is inconsistent, the three estimations are not equal. The power method of squaring the matrix takes the sum of all the three estimations, taking into account direct and indirect estimations.

When matrices are inconsistent, the ratio between two priorities may differ from our direct estimations. This phenomenon criticised from Bana e Costa, et 
al. (2001) is due to the consideration of indirect estimations in the priorities calculation. As indirect estimations contain important information, we think that their influence on the final priorities must be taken into account. Therefore we do not consider the criticism from Bana e Costa, et al. (2001) to be valid. A debate has been opened on the weight to be allocated on the indirect estimations (Brugha, 2000).

\subsection{The geometric mean}

In this approach, the priorities are given by the geometric mean (example 4), which minimizes the logarithmic error (Crawford, 1985):

$$
\begin{gathered}
\sum_{i=1}^{n} \sum_{j=1}^{n}\left(\ln \left(a_{i j}\right)-\ln \left(\frac{p_{i}}{p_{j}}\right)\right)^{2} \text { where } a_{i j} \text { is the comparison between } i \text { and } j \\
p_{i} \text { is the priority of } i
\end{gathered}
$$

This method is insensitive to an inversion of the scale: the geometric mean of the rows and the columns give the same ranking.

Saaty (1990) criticizes this method because he sees no conceptual justification for working with a logarithmic scale. He adds that the calculation is made only with a row, i.e. the indirect estimations are not considered (Saaty 1984a, 1984b).

Example 4:

The priorities from the matrix of the example 1 calculated with the geometric mean are:

$p_{1}=\sqrt[3]{1 \cdot 6 \cdot 3}=2.62, p_{2}=\sqrt[3]{\frac{1}{6} \cdot 1 \cdot \frac{1}{2}}=0.44, p_{3}=\sqrt[3]{\frac{1}{3} \cdot 2 \cdot 1}=0.87$

Normalizing, we obtain: $\vec{p}=(0.67,0.11,0.22)$

\section{Simulations}

We generated matrices of different dimensions and inconsistencies in a Monte Carlo simulation and compared the results of the four derivation methods.

Budescu et al. (1986) and Triantaphyllou et al. (1990) generate random matrices and then assign them to an inconsistency group. We do not think that a random filling of matrices reflects the process used by decision makers when using AHP. Additionally we expect the rate of unusable matrices (totally inconsistent matrices) to be very high. 
Golany et al. (1993) have improved the matrix generation process. Only the first row is randomly selected from a uniform distribution in the interval $[1,9]$. The other comparisons $a_{i j}$ are randomly selected from the distribution $a_{i j}$ $\in\left[\frac{(100-k) \cdot a_{1 j}}{100 \cdot a_{1 i}}, \frac{(100+k) \cdot a_{1 j}}{100 \cdot a_{1 i}}\right]$, where $j>i>1$ and $\mathrm{k} \in[10,20, \ldots, 90]$. This method considers the errors of the decision maker to be multiplicative: $a_{i j}$. $e_{i j}$, where $e_{i j}$ is the error factor. Multiplicative errors consider low values to be less perturbation sensitive than high values. For example, if we have an error of $e$ $=2$, the initial comparison 2 is subject to a shift of $2=(4 \cdot 2)-2$ units. For the same error, the comparison 4 is subject to a shift of $4=(4 \cdot 2)-4$ units.

This method suffers from other problems: The first row contains discrete values from the interval [1,9], but the other entries obtain continuous values which could be even values outside the comparisons scale!

Our simulation approach uses an additive error of $a_{i j}+e_{i j}$. Additive errors modify all values of the comparison scale equally. All matrix values come from a discrete interval $[1,9]$ and we discuss how to deal the extremities of the scale.

\subsection{Description}

The experiment is based on five steps:

\section{1) Building inconsistency groups}

For dimensions from three to seven, we build five groups of inconsistent matrices based on the consistency ratios $([0,0.02[;[0.02,0.04[;[0.04,0.06[$; $[0.06,0.08[;[0.08,0.1[)$. We have chosen consistency ratios smaller than 0.1 (Saaty's limit to accept matrices as "near consistent"). For each five different dimensions and each five consistency group, one hundred matrices have been generated, which results in a total of $100 \cdot 5 \cdot 5=2500$ matrices.

\section{2) Generating a consistent matrix}

A consistent matrix is constructed in four steps (Ishizaka, et al., 2004a): the principal diagonal, the independent, the transitive and the reciprocal comparisons. Only the first diagonal below the main diagonal is randomly selected from the fundamental scale values $[1 / 9,1 / 8, \ldots, 1,2, \ldots, 9]$, the other comparisons can be deduced by the transitivity and the reciprocity rules. If a value is induced outside the fundamental scale, the matrix is rejected.

\section{3) Introducing impurities}

The number of impurities introduced in the upper part of the matrix is randomly selected from the interval $\left[0,1, \ldots, \frac{n^{2}-n}{2}\right]$, where $n$ is the dimension of the matrix. This impurity is represented as a shift in the comparison scale (equivalent to an additive error term) reaching at most $45 \%$ of the extreme pref- 
erence 9, i.e \pm 4 scale positions (example 5). The comparisons modified by slight perturbations (impurities) are chosen randomly. The process is stochastic; therefore a comparison can be modified by more than one perturbation. The amplitude of the maximum shift allowed is a delicate question. The introduced error should reflect the inconsistencies of a typical decision maker. It should not be too small to exclude possible scenarios. In this paper we assume that a maximum shift of 4 units meets the requirements. Anyway, if the impurities are too high and induce strong inconsistencies (i.e. a consistency ratio of more than 0.1 ) then the matrix is rejected.

The percentage of each new value is equal to $100 / 9=11.11 \%$, where the denominator 9 is the number of possible new values. If the original comparison value is greater than 5 or smaller than $1 / 6$ then the number of shift possibilities is less than 9 .

\section{Example 5:}

If the consistent comparison is 3 , a shift from at most 4 positions scale is admitted. The new value can be between $1 / 3$ and 7 .

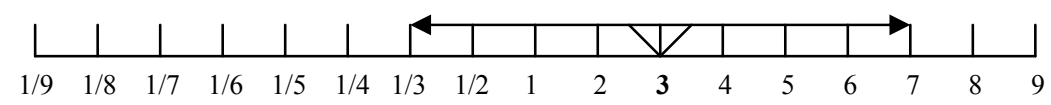

One question which arises from this is whether the decision maker is subject to a border effect and which is the impact? Two contradictory theories exist:

- The extreme value is used more frequently than others. Every time the user wishes to enter a value outside the scale, the highest authorised value will be chosen. For example, if a decision maker places $a_{i j}=5$ and $a_{j k}=4$ then $a_{i k}=9$ will probably be entered.

- Psychologist have argued that extreme values are less used than middle values (e.g. Berekoven, et al., 2001).

We suppose that these tendencies compensate for each other and no border effect exists. To avoid a border effect, the percentage of each new value remains $11.11 \%$ and the original comparison gets the remaining percentage. For example, if the entry is 8 , the new value can be $9,7,6,5$, or 4 with a probability of $11.11 \%$ for each number and 8 with a probability of $100-(5 \cdot 11.11)=44.45 \%$.

\section{4) Assigning the matrix to an inconsistency group}

The consistency ratio (C.R.) is calculated and the matrix is classified into one of the five inconsistency groups (see step 1) or rejected if the C.R. is equal or higher than 0.1 .

\section{5) Deriving the priorities}

The priorities are calculated with the following four methods: 
- Mean of the Normalised Values (MNV)

- Right Eigenvalue Method (REM)

- Left Eigenvalue Method (LEM)

- Geometric Mean (GM)

The solutions are collected and analysed. The results are shown in the next paragraph.

\section{Results}

\subsection{Rankings contradictions}

The four derivation methods do not always result in the same ranking. The number of ranking contradictions out of 100 with regard to the dimension of the matrix and the consistency ratio is represented in table 1.

\begin{tabular}{|l|c|c|c|c|c||c|}
\hline & $\operatorname{dim} \mathbf{3}$ & $\operatorname{dim} \mathbf{4}$ & $\operatorname{dim} \mathbf{5}$ & $\operatorname{dim} \mathbf{6}$ & $\operatorname{dim} \mathbf{7}$ & total \\
\hline C.R. $<\mathbf{0 . 0 2}$ & 0 & 0 & 7 & 8 & 12 & 27 \\
\hline C.R. $<\mathbf{0 . 0 4}$ & 0 & 5 & 15 & 17 & 30 & 67 \\
\hline C.R. $<\mathbf{0 . 0 6}$ & 0 & 8 & 20 & 23 & 43 & 94 \\
\hline C.R. $<\mathbf{0 . 0 8}$ & 0 & 15 & 28 & 43 & 58 & 144 \\
\hline C.R. $<\mathbf{0 . 1 0}$ & 0 & 20 & 33 & 58 & 81 & 192 \\
\hline \hline total & 0 & 48 & 103 & 149 & 224 & \multicolumn{1}{|c}{} \\
\cline { 1 - 5 } & & & &
\end{tabular}

Table 1: Number of ranking contradictions with regard to the dimension of the matrix and the consistency ratio

All methods provide the same ranking for matrices of dimension three. The ranking contradiction phenomenon increases linearly with the inconsistencies (figure 3 ) and the dimension of the matrix (figure 4). This is easily explicable: if the number of alternatives increases, the possibilities of reversal rise too. The process is analogous to the disorder measured by the consistency ratio: when it increases, the probabilities of reversal are higher. Tables 2 and 3 show that the linear correlation between the data is very high. 


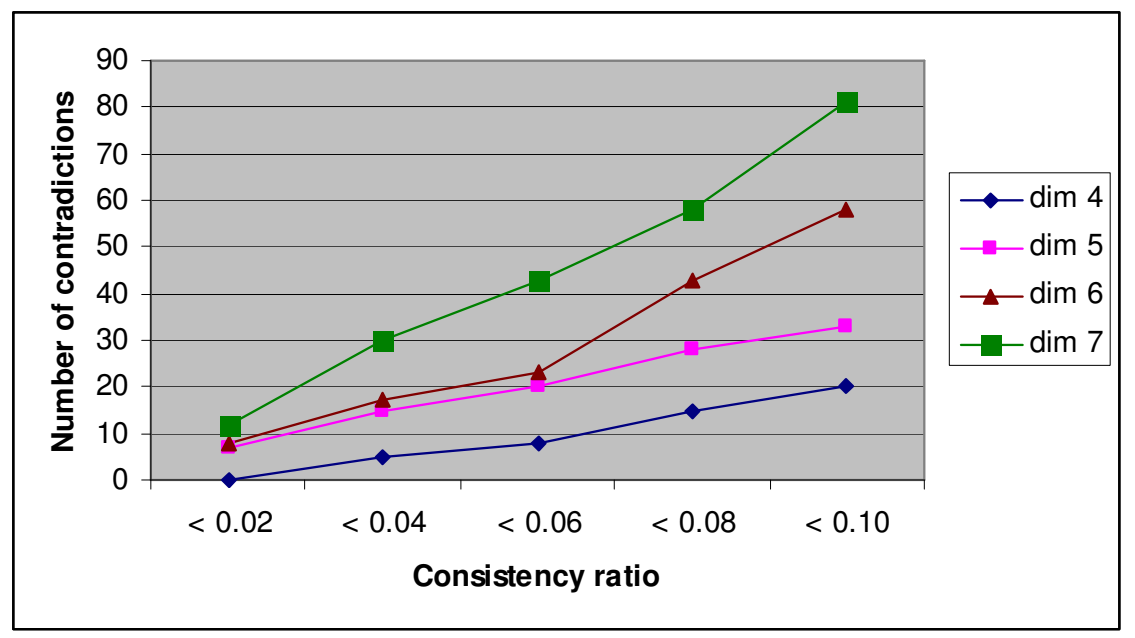

Figure 3: Number of rankings contradictions in function of the consistency ratio

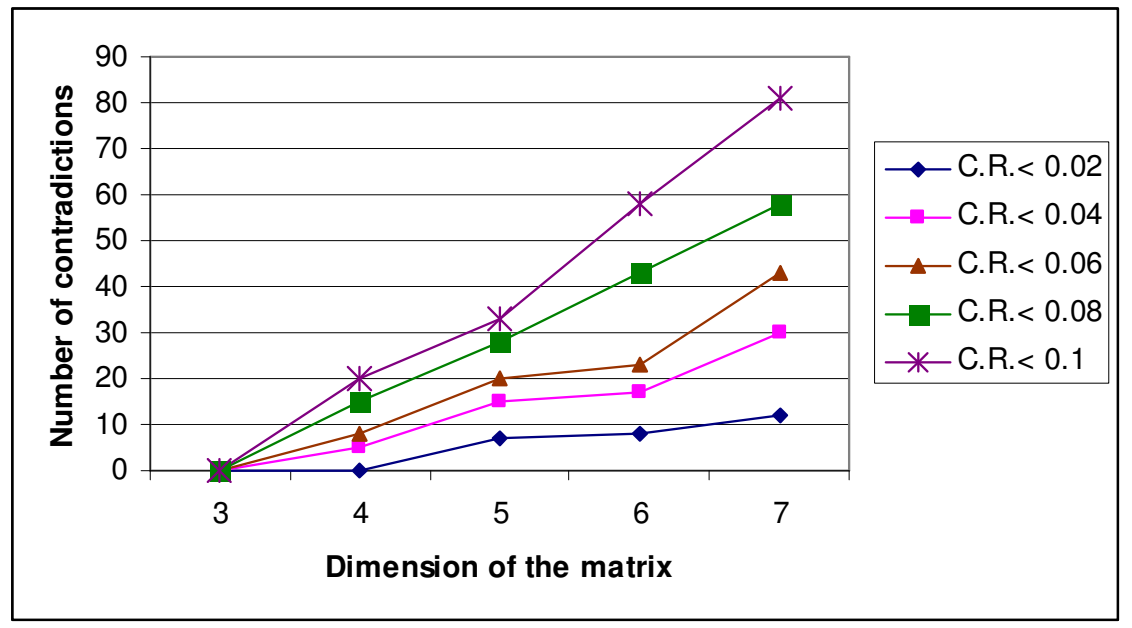

Figure 4: Number of rankings contradictions in function of the dimension of the matrix

\begin{tabular}{|c|c|c|}
\hline $\begin{array}{c}\text { Dimension of } \\
\text { the matrix }\end{array}$ & Slope & Correlation \\
\hline 4 & 5 & 0.99 \\
\hline 5 & 6.5 & 0.99 \\
\hline 6 & 12.6 & 0.98 \\
\hline 7 & 16.6 & 0.99 \\
\hline
\end{tabular}

Table 2: Slope of the linear functions "consistency ratio" to "number of contradictions" and the correlation between these two set of data 


\begin{tabular}{|c|c|c|}
\hline Consistency ratio & Slope & Correlation \\
\hline$<0.02$ & 3.2 & 0.96 \\
\hline$<0.04$ & 7.2 & 0.98 \\
\hline$<0.06$ & 10.1 & 0.97 \\
\hline$<0.08$ & 14.4 & 0.99 \\
\hline$<0.1$ & 20 & 0.99 \\
\hline
\end{tabular}

Table 3: Slope of the linear functions "dimension of the matrix" to "number of contradictions" and the correlation between these two set of data

\subsection{Comparisons between alternative methods}

The highest number of ranking contradictions occurs between the right eigenvalue method (REM) and the left eigenvalue method (LEM) followed by the the left eigenvalue method (LEM) and the mean of the normalised values (MNV) (table 4). Relatively few ranking contradictions are found between the right eigenvalue method (REM) and the mean of the normalised values (MNV). This probably explains why the mean of the normalised values is widely used as an approximate method of the eigenvalue method.

\begin{tabular}{|l|c|c|c|}
\hline$[\%]$ & REM & LEM & GM \\
\hline MNV & 9 & 23 & 17 \\
\hline REM & - & 25 & 18 \\
\hline LEM & - & - & 19 \\
\hline
\end{tabular}

Table 4: Percent of ranking contradiction between each method

A $\chi^{2}$ test (table 5) confirms our observations of table 4 . The levels of contradiction are similar if $\chi^{2}<3.841$ (significance level at $\mathrm{P}\left(\chi^{2}\right)=0.05$ ). Therefore the number of contradictions between the methods MNV - REM is significantly lower than for the others comparisons and the number of contradictions between the methods MNV - LEM and REM - LEM is significantly higher.

\begin{tabular}{|c|c|c|c|c|c|}
\hline$\chi^{2}$ & $\begin{array}{c}\text { MNV/ } \\
\text { LEM } \\
\mathbf{( 5 7 3 )}\end{array}$ & $\begin{array}{c}\text { MNV/ } \\
\text { GM } \\
\mathbf{( 4 3 1 )}\end{array}$ & $\begin{array}{c}\text { REM/ } \\
\text { LEM } \\
\mathbf{( 6 1 8 )}\end{array}$ & $\begin{array}{c}\text { REM/ } \\
\text { GM } \\
\mathbf{( 4 4 9 )}\end{array}$ & $\begin{array}{c}\text { LEM/ } \\
\text { GM } \\
\mathbf{( 4 7 5 )}\end{array}$ \\
\hline $\begin{array}{c}\text { MNV/ } \\
\text { REM (228) }\end{array}$ & 176.9 & 72 & 216.4 & 83.4 & 101.0 \\
\hline $\begin{array}{c}\text { MNV/ } \\
\text { LEM (573) }\end{array}$ & - & 25.1 & $\mathbf{2 . 2}$ & 18.9 & 11.6 \\
\hline $\begin{array}{c}\text { MNV/ } \\
\text { GM (431) }\end{array}$ & - & - & 42.2 & $\mathbf{0 . 4}$ & $\mathbf{2 . 6}$ \\
\hline $\begin{array}{c}\text { REM/ } \\
\text { LEM (618) }\end{array}$ & - & - & - & 34.0 & 23.9 \\
\hline $\begin{array}{c}\text { REM/ } \\
\text { GM (449) }\end{array}$ & - & - & - & - & $\mathbf{0 . 9}$ \\
\hline
\end{tabular}

Table 5: $\chi^{2}$ test for the number of contradictions (in brackets) between the methods; if $\chi^{2}<$ 3.841 (significance level at $\mathrm{P}\left(\chi^{2}\right)=0.05$ ), contradiction levels are similar (in bold) 


\subsection{Reversed priorities}

Table 6 represents the mean difference between two reversed priorities. We illustrate the difference between two reversed priorities with the example 6 .

\begin{tabular}{|l|l|l|l|l|}
\hline & \multicolumn{1}{|c|}{ MNV } & \multicolumn{1}{|c|}{ REM } & \multicolumn{1}{|c|}{ LEM } & \multicolumn{1}{|c|}{ GM } \\
\hline \multirow{2}{*}{ Dim 4 } & $0.008 \pm$ & $0.008 \pm$ & $0.020 \pm$ & $0.007 \pm$ \\
& 0.000 & 0.000 & 0.001 & 0.000 \\
\hline \multirow{2}{*}{ Dim 5 } & $0.007 \pm$ & $0.009 \pm$ & $0.016 \pm$ & $0.007 \pm$ \\
& 0.000 & 0.000 & 0.000 & 0.000 \\
\hline \multirow{2}{*}{ Dim 6 } & $0.013 \pm$ & $0.017 \pm$ & $0.017 \pm$ & $0.012 \pm$ \\
& 0.000 & 0.001 & 0.001 & 0.000 \\
\hline \multirow{2}{*}{ Dim 7 } & $0.007 \pm$ & $0.011 \pm$ & $0.012 \pm$ & $0.007 \pm$ \\
& 0.000 & 0.000 & 0.000 & 0.000 \\
\hline
\end{tabular}

Table 6: Mean difference between two reversed priorities classified by dimension and method

Example 6:

The priorities are calculated with method $\mathrm{A}$ and $\mathrm{B}$ : priorities of method $\mathrm{A}=$ $(0.244 ; 0.439 ; 0.11 ; 0.101 ; 0.106)$ and priorities of method $\mathrm{B}=(0.25 ; 0.455$; $0.104 ; 0.098 ; 0.094)$. A contradiction arises between ranks 4 and 5 . The difference between the two reversed priorities is for the method A: $0.106-0.101=$ $\mathbf{0 . 0 0 5}$ and for the method B: $0.098-0.094=\mathbf{0 . 0 0 4}$.

These differences are small $(\leq 0.020)$ as we can confirm with a binomial test. If we have 4 priorities, the equivalent distance between them is of 0.2 (figure 5).

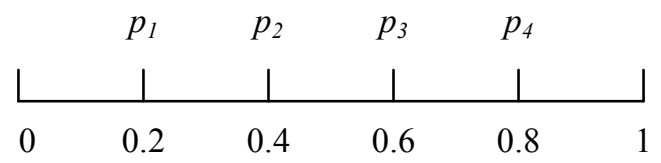

Figure 5: The equivalent distance between the four priorities $p_{i}$ is 0.2

The zero-hypothesis states that if we randomly set the priorities on the scale 0 to 1 , the interval between them is in $50 \%$ of the cases higher than 0.2 and in the others $50 \%$ lower than 0.2 . In our experiment, we can reject the zero-hypothesis because in no cases the difference between two reserved priorities is higher than 0.2 .

The same binomial test can be repeated with the matrices of the higher dimensions. Again the zero-hypothesis is rejected because no difference between two reserved priorities is higher than the distance of an equivalent distance between the priorities. 


\section{Conclusion}

In a Monte Carlo simulation, consistent matrices of different dimensions have been generated and an additive error has been introduced randomly. Then four priority derivation methods have been compared. We have not observed any classification difference for matrices of dimension three and for perfect consistent matrices. For superior dimensions the differences between the solutions of derivation methods are minor. This applies even to the mean of normalized values where no mathematical theory for inconsistent matrices exists. Only very close priorities suffer from ranking contradictions. The number of contradictions increases with the inconsistencies and the matrix dimension. Ranking contradictions are issued from the different processing methods of inconsistencies, the more inconsistencies, the more contradictions. The eigenvalue approach considers the indirect estimations in the calculation of priorities. The calculated priorities depend on how much these comparisons are intransitive. The geometric mean uses only the direct comparisons. For this reason the geometric mean of the columns and the geometric mean of the rows always give the same ranking. Contradictions in the ranks can be used as an indicator to decrease the inconsistency in order to determine better the dominance of close priorities. Decreasing the dimension with clusters could be also used in case of comparison of heteroclite alternatives (Saaty, 2001b; Ishizaka, 2004b). This technique allows an easier comparison and an extension of the comparison scale from 1-9 to 1- $\infty$.

There is a high level of agreement between the different scaling techniques. This has already been observed in previous studies, where the generation techniques of matrices for the simulation were different: totally randomly filling of the matrices (Budescu, et al., 1986) and consistent construction of the matrices followed with an introduction of a randomly multiplicative error (Golany, et al., 1993). We do not think that one method is superior to another. We advice decision-makers also to consider other criteria like "easy to use" in selecting of their derivation method.

The concordance of the right and left eigenvalue is the lowest compared to any other pair of solutions. This finding reinforces the warning of Johnson et al. (1979) regarding the use of the right eigenvector.

This study should help decision maker with their choice of a priorities derivation method. To understand the priority derivation process, we have also developed an intelligent tutoring system (Ishizaka, et al., 2003). It guides the user in the construction of a consistent or a near consistent matrix and teaches how to derive priorities by the four methods and to observe the effects of slight perturbations on a consistent matrix. All exercises allow the user to freely navigate through the solution trees.

\section{References}

Bana e Costa C. A., Vansnick J.-C. (2001) A Fundamental Criticism to Saaty's Use of the Eigenvalue Procedure to Derive Priorities, Working Paper LSEOR 01.42; retrieved December 2006 from http://www.lse.ac.uk/collections/operationalResearch/research/ 
workingPapers.htm

Barzilai J., Cook W.D., Golany B. (1987) Consistent Weights for Judgements Matrices of the Relative Importance of Alternatives. Operations Research Letters 6,1: 131-134

Barzilai J., Golany B. (1990) Deriving Weights from Pairwise Comparison Matrices: the Additive Case. Operations Research Letters 9: 407-10

Barzilai J. (1997) Deriving Weights from Pairwise Comparison Matrices, Journal of the Operational Research Society (JORS) 48,12: 1226-32

Barzilai J. (2001a) Notes on the Analytic Hierarchy Process, Proceedings of the NSF Design and Manufacturing Research Conference: 1-6

Barzilai J. (2001b) Basic Principles of Measurement, Proceedings of the IEEE International Conference on Systems, Man and Cybernetics: 395-400

Barzilai J. (2002) Notes on Measurement and Decision Theory, Proceedings of the NSF Design and Manufacturing Research Conference: 1-11

Berekoven L., Eckert W., Ellenrieder P. (2001) Marktforschung. Methodische Grundlagen und praktische Anwendung, 9. Auflage, Gabler Verlag, Wiesbaden

Blankmeyer E. (1987) Approaches to Consistency Adjustments, Journal of Optimization Theory and Applications 45: 479-88

Brugha C. (2000) Relative Measurement and the Power Function, European Journal of Operational Research 121, 3: 627-640

Budescu D.V., Zwick R., Rapoport A. (1986) A Comparison of the Eigenvalue Method and the Geometric Mean Procedure for Ratio Scaling, Applied Psychological Measurement 10, 1: 69-78

Chu A.T.W., Kalabra R.E., Spingarn K.A. (1979) A Comparison of Two Methods for Determining the Weights of Belonging to Fuzzy Sets, Journal of Optimization Theory and Applications 27: 531-538

Cogger K.O., Yu P.L. (1985) Eigenweight Vectors and Least Distance Approximation for Revealed Preference in Pairwise Weight Ratios, Journal of Optimization Theory and Applications 46: 483-491

Cook W.D., Kress M. (1988) Deriving Weights from Pairwise Comparison Ratio Matrices: An Axiomatic Approach, European Journal of Operational Research 37: 355-62

Crawford G., Williams C. (1985) A Note on the Analysis of Subjective Judgement Matrices, Journal of Mathematical Psychology 29: 387-405

Golany B., Kress M. (1993) A Multicriteria Evaluation of the Methods for Obtaining Weights from Ratio-Scale Matrices, European Journal of Operational Research 69: 210-202

Golden B.L., Wasil E.A., Harker P.T. (1989) The analytic hierarchy process: applications and Studies, Springer-Verlag, Heidelberg

Ishizaka A., Lusti M. (2003) An Intelligent Tutorial System for AHP. In: K. Šorić, T. Hunjak, R. Scitovski (eds.), Proceedings of the $9^{\text {th }}$ International Conference on Operational Research KOI 2002: 215-223

Ishizaka A., Lusti M. (2004a) An Expert Module to Improve the Consistency of AHP Matrices, International Transactions in Operational Research 11, 1: 97-105

Ishizaka A. (2004b) The Advantages of Clusters in AHP, 15th Mini-Euro Conference, MUDSM

Jensen R.E. (1984) An Alternative Scaling Method for Priorities in Hierarchical Structures, Journal of Mathematical Psychology 28, 3: 317-332

Johnson C.R., Beine W.B., Wang T.Y. (1979) Right-left asymmetry in an eigenvector ranking procedure, Journal of Mathematical Psychology 18: 61-64

Harker P.T., Vargas L. (1987) The Theory of Ratio Scale Estimation: Saaty's Analytic Hierarchy Process, Management Science 33,11: 1383-1403

Lusti M. (2002) Data Warehousing und Data Mining, 2nd edn. Springer-Verlag, Berlin

Saaty Th. L. (1977) A Scaling Method for Priorities in Hierarchical Structures, Journal of Mathematical Psychology 15: 234-281 
Saaty Th. L. (1980) The Analytic Hierarchy Process, Mac Gray-Hill, New York

Saaty Th. L., Vargas L. G. (1984a) Inconsistency and Rank Preservation, Journal of Mathematical Psychology 28: 205-214

Saaty Th. L., Vargas L. G. (1984b) Comparison of Eigenvalue, Logarithmic Least Squares and Least Squares Methods in Estimating Ratios, Mathematical Modelling 5: 309-324

Saaty Th. L. (2001a) Decision-making with the AHP: Why is the Principal Eigenvector necessary? Proceedings of the Sixth International Symposium on the Analytic Hierarchy Process (ISAHP 2001): 383-396

Saaty Th. L. (2001b) The Seven Pillars of the Analytic Hierarchy Process. In: Köksalan, M. et al. (eds). Multiple Criteria Decision Making in the New Millennium. Proceedings of the 15th International Conference, MCDM, Springer, Lect. Notes Econ. Math. Syst. 507, Berlin: 15-37

Saaty Th. L. (2003) Decision-making with the AHP: Why is the Principal Eigenvector necessary? European Journal of Operational Research 145: 85-91

Salo A. A., Hämäläinen R.P. (1997), On the Measurement of Preferences in the Analytic Hierarchy Process, Journal of Multi-Criteria Decision Analysis, 6: 309-319

Shim J.P. (1989), Bibliography Research on the Analytic Hierarchy Process (AHP), Socio-Economic Planning Sciences, 23:161-167

Takeda E., Cooger K.O., Yu P.L. (1987) Estimating Criterion Weights Using Eigenvectors: a Comparative Study. European Journal of Operational Research 29: 360-369

Triantaphyllou E., Pardalos P.M., Mann S.H. (1990) A Minimization Approach to Membership Evaluation in Fuzzy Sets and Error Analysis, Journal of Optimization Theory and Applications 66, 2: 275-287

Vargas L.G. (1990) An Overview of the Analytic Hierarchy Process and its Applications, European journal of operations research 48, 1:2-8

Zahedi F., The analytic hierarchy process: a survey of the method and its applications, Interface 16, 96-108, 1986 